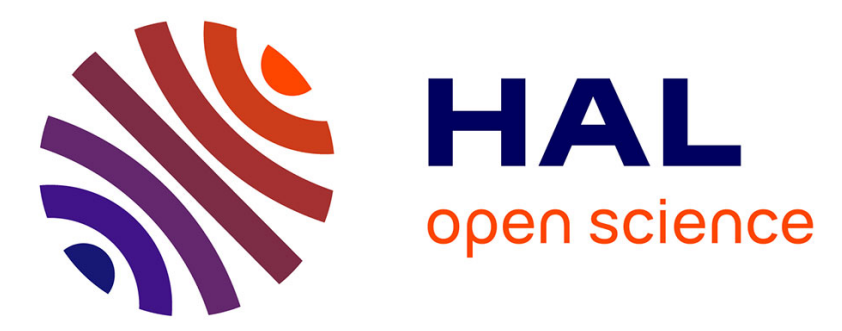

\title{
Seventeen new exon-primed intron-crossing polymerase chain reaction amplifiable introns in fish
}

\author{
M. Hassan, C. Lemaire, Cécile Fauvelot, F. Bonhomme
}

\section{To cite this version:}

M. Hassan, C. Lemaire, Cécile Fauvelot, F. Bonhomme. Seventeen new exon-primed intron-crossing polymerase chain reaction amplifiable introns in fish. Molecular Ecology Notes, 2002, 10.1046/j.14718286 . ird- 03044170

\author{
HAL Id: ird-03044170 \\ https://hal.ird.fr/ird-03044170
}

Submitted on 7 Dec 2020

HAL is a multi-disciplinary open access archive for the deposit and dissemination of scientific research documents, whether they are published or not. The documents may come from teaching and research institutions in France or abroad, or from public or private research centers.
L'archive ouverte pluridisciplinaire HAL, est destinée au dépôt et à la diffusion de documents scientifiques de niveau recherche, publiés ou non, émanant des établissements d'enseignement et de recherche français ou étrangers, des laboratoires publics ou privés. 


\title{
PRIMER NOTE \\ Seventeen new exon-primed intron-crossing polymerase chain reaction amplifiable introns in fish
}

\author{
M. HASSAN, * C. LEMAIRE, ${ }^{*}$ C. FAUVELOT*† and F. BONHOMME* \\ *Laboratoire Génome, Populations, Interactions Cnrs Umr 5000, SMEL 1, Quai de la Daurade, 34200 Sète, France; †cole Pratique, \\ Des Hautes Etudes, Université de Perpignan, Perpignan, France
}

\begin{abstract}
We used exon-primed, intron-crossing polymerase chain reaction (EPIC-PCR) amplification to assay variation in nuclear loci in some teleost fishes (Carangidae, Centropomidae, Chaetodontidae, Clupeidae, Holocentridae, Moronidae, Mullidae, Pomacentridae, Scombridae, Siganidae). We designed primers in the conserved regions flanking splice sites of consecutive exons of different genes, allowing the amplification of 17 putative introns. Among the satisfactory amplified systems, 14 showed length polymorphism with 2-14 alleles.
\end{abstract}

Keywords: EPIC-PCR, length polymorphism, teleost fishes

Received 27 February 2002; revision received 10 April 2002; accepted 10 April 2002

Introns range in size from $\approx 80$ to 10000 or more nucleotides. Unlike exons, the nucleotide sequence of introns seems to be little constrained; it is often possible to alter most of their sequence without greatly affecting gene function. Introns accumulate mutations rapidly and often display a high genetic variability, including length polymorphism (Palumbi 1995). Exon-primed, intron-crossing polymerase chain reaction (EPIC-PCR) amplification provides a widely applicable strategy for finding DNA polymorphism in eukaryotic genomes. Thus, introns can be targeted as markers of population variation and subdivision (Lessa 1992). They constitute suitable markers for analysing population structure within a species as well as for reconstructing relationships among closely related species (He \& Haymer 1997). Intron variation allows high-resolution genetic characterization of invading populations in both natural and managed systems (Villablanca et al. 1998). This method was used to detect and rapidly assess allelic variation at the nucleotide level in mammals (Palumbi \& Baker 1994), birds (Heslewood et al. 1998), insects (Gomulski et al. 1998; He \& Haymer 1999), crustaceans (Bierne et al. 2000) and mussels (Côrte-Real et al. 1994; Daguin et al. 2001).

Using the same approach, we designed primers in the conserved regions of consecutive exons of different genes, after a search of exonic sequences published to date (Table 1) in different species of teleost fishes. Each $10 \mu \mathrm{L}$ PCR contained

Correspondence: M. Hassan. Fax: +33 4674633 99; E-mail: m-hassan@crit.univ-montp2.fr
0.5 U of Taq DNA polymerase (Promega, Madison WI, USA), $1 \mu \mathrm{L}$ buffer (Promega), $0.75 \mathrm{~mm}$ dNTPs, $1.8 \mathrm{~mm} \mathrm{MgCl}_{2}$ and 30-50 ng template DNA. Right before the PCR, primer (Forward) was labelled radioactively with the ${ }^{33} \mathrm{P}$ using $1 \mathrm{U}$ of polynucleotide-kinase T4 (Eurogenetic, Liège, Belgium, $10 \mathrm{U} / \mu \mathrm{L}$ ) in a reaction mixture contained $0.3 \mu \mathrm{M}$ of the primer and $0.4 \mathrm{mCi} / \mathrm{mL}$ of $\left[\gamma^{33} \mathrm{P}\right]$ ATP (Amersham Pharmacia Biotech, UK). This mixture was incubated $30 \mathrm{~min}$ at $37^{\circ} \mathrm{C}$. PCR was performed in a Crocodile III Thermocycler (Appligène, Strasbourg, France), using the following cycle profile: one initial denaturation step at $95^{\circ} \mathrm{C}$ for $3 \mathrm{~min}$, followed by 35 cycles of amplification [denaturation at $94{ }^{\circ} \mathrm{C}$ for $12 \mathrm{~s}$, annealing at (see Table 2 ) for $12 \mathrm{~s}$ and extension at $72{ }^{\circ} \mathrm{C}$ for $20 \mathrm{~s}$ ] and a final extension at $72{ }^{\circ} \mathrm{C}$ for $5 \mathrm{~min}$. PCR products were mixed with $5 \mu \mathrm{L}$ of formamide dye (95\% formamide, $20 \mathrm{~mm}$ EDTA, $0.05 \%$ xylene cyanol and $0.05 \%$ bromophenol blue), denaturated at $95^{\circ} \mathrm{C}$ for $5 \mathrm{~min}$ and $4 \mu \mathrm{L}$ were loaded on a $6 \%$ polyacrylamide gel in TBE buffer. The gels were run for $3 \mathrm{~h}$ at $50 \mathrm{~W}$. After drying, the gels were exposed against films of Biomax autoradiograph.

Table 2 presents the principal results of the 17 EPIC-PCR on the tested species. The simultaneous amplification of two or three loci can reveal either the presence of another gene, or of a pseudogene in the species that show these multiple amplifications. It does not seem that these simultaneous amplifications disturb the quality of PCRs and the reading of the genotypes because: (i) the supplementary profiles do not present parasitic bands, (ii) the variation 


\begin{tabular}{|c|c|c|c|c|}
\hline Gene & Intron number & Intron name & Primer name & Primer sequence $\left(5^{\prime}-3^{\prime}\right)$ \\
\hline \multirow[t]{2}{*}{ Creatin kinase (mitochondrial) } & \multirow[t]{2}{*}{ intron 7} & \multirow[t]{2}{*}{ Ck7 } & Ck7F & AAGAGGGTCTTTGACAGGTTCTGC \\
\hline & & & Ck8R & TTCTCCTGGATCAGACGCTCCACC \\
\hline \multirow[t]{2}{*}{ Glyceraldehyde-3-phosphate dehydrogenase } & \multirow[t]{2}{*}{ intron 2} & \multirow[t]{2}{*}{ Gpd2 } & Gpd2F & GCCATCAATGACCCCTTCATCG \\
\hline & & & Gpd3R & TTGACCTCACCCTTGAAGCGGCCG \\
\hline \multirow[t]{4}{*}{ Growth hormone } & \multirow[t]{2}{*}{ intron 2} & \multirow[t]{2}{*}{ Gh2 } & Gh2F & AGCGTTTCTCCATTGCCGTCAGC \\
\hline & & & Gh3R & TCTTGTTGAGTTGACGCTGGTCC \\
\hline & \multirow[t]{2}{*}{ intron 5} & \multirow[t]{2}{*}{ Gh5 } & Gh5F & AGGCCAATCAGGACGGAGC \\
\hline & & & Gh6R & TGCCACTGTCAGATAAGTCTCC \\
\hline \multirow[t]{2}{*}{ Aldolase C } & \multirow[t]{2}{*}{ intron 1} & \multirow[t]{2}{*}{ AldoC1 } & Aldoc1F & CCTGGCTGCGGACGAGTCTGTGGG \\
\hline & & & Aldoc2R & GGCGGTACTGTCTGCGGTTCTCC \\
\hline \multirow[t]{8}{*}{ Aldolase B } & \multirow[t]{2}{*}{ intron 1} & \multirow[t]{2}{*}{ AldoB1 } & Aldob1-1F & GCTCCAGGAAAGGGAATCCTGGC \\
\hline & & & Aldob1-2R & CTCGTGGAAGAAGATGATCCCGCC \\
\hline & \multirow[t]{2}{*}{ intron 2} & \multirow[t]{2}{*}{ AldoB2 } & Aldob2F & TCAGGGCATTGTCGTCGG \\
\hline & & & Aldexon3R & CTGTTCCATTTAGACCAGC \\
\hline & \multirow[t]{2}{*}{ intron 4} & \multirow[t]{2}{*}{ AldoB4 } & Aldo5F & GCCAGATATGCCAGCATCTGCC \\
\hline & & & Aldo3.1R & GGGTTCCATCAGGCAGGATCTCTGGC \\
\hline & \multirow[t]{2}{*}{ intron 5} & \multirow[t]{2}{*}{ AldoB5 } & Aldo3F & TCCTGCCTGATGGAACCC \\
\hline & & & Aldo2R & CAGGTACACATGGTGGTC \\
\hline \multirow[t]{6}{*}{ Gonadotropin-releasing hormone 3} & \multirow[t]{2}{*}{ intron 1} & \multirow[t]{2}{*}{ GnRH3-1 } & GnRH1F & AATGCACCACATGCTAACAAGGC \\
\hline & & & GnRH1R & CGCACCATCACTCTGCTGTTCGC \\
\hline & \multirow[t]{2}{*}{ intron 2} & \multirow[t]{2}{*}{ GnRH3-2 } & GnRH2F & AGAAGTGTGGGAGAGCTAGAGGC \\
\hline & & & GnRH2R & AGAGACACCACTTCTCCTGTACCC \\
\hline & \multirow[t]{2}{*}{ intron 3} & GnRH3-3 & GnRH3F & GCCCAAACCCAAGAGAGACTTAGACC \\
\hline & & & GnRH3R & TTCGGTCAAAATGACTGGAATCATC \\
\hline Alpha amylase & intron 1 & Am2B-1 & Am2b1F & CCTTCATCTTCCAGGAGGTAC \\
\hline & & & $\mathrm{Am} 2 \mathrm{~b} 2 \mathrm{R}$ & TTCACCTCCCAGATCAATAAC \\
\hline & intron 2 & Am2B-2 & $\mathrm{Am} 2 \mathrm{~b} 2 \mathrm{~F}$ & GGCGATAAGTTGTCTTACACC \\
\hline & & & $\mathrm{Am} 2 \mathrm{~b} 3 \mathrm{R}$ & AGCCCTCTCCCCAGTTCCTGC \\
\hline & intron 3 & Am2B-3 & Am2b3F & TGGAACCGAAACATTGTGAAC \\
\hline & & & Am2b4R & CCCATCCAGTCATTCTGATCC \\
\hline Alpha tropomyosin & intron 1 & $\operatorname{Tr} 1$ & $\operatorname{Tr} 1 \mathrm{~F}$ & AGGGAACAGAGGATGAGCTGGAC \\
\hline & & & $\operatorname{Tr} 1 \mathrm{R}$ & TCTCAGCTTCCTCCAGCTTGGTG \\
\hline Major histocomptability complex class II antigen & intron 1 & MhcII & Mhc1F & ACTCTAATCTGGAGTACATGC \\
\hline & & & Mhc2R & CAGGAGATCTTCTCTCCAGCC \\
\hline
\end{tabular}


Table 2 Details of general results

\begin{tabular}{|c|c|c|c|c|c|c|c|c|c|c|}
\hline Intron name & Species name & $\begin{array}{l}\text { Sample } \\
\text { size (ind.) }\end{array}$ & $\begin{array}{l}\text { No. of } \\
\text { amplified } \\
\text { loci }\end{array}$ & $\begin{array}{l}\text { Annealing } \\
\text { temp. }\left({ }^{\circ} \mathrm{C}\right)\end{array}$ & $\begin{array}{l}\text { Name of } \\
\text { amplified } \\
\text { loci }\end{array}$ & $\begin{array}{l}\text { Length } \\
\text { polymorphism }\end{array}$ & $\begin{array}{l}\text { No. of } \\
\text { alleles at } \\
\text { each locus }\end{array}$ & $H_{\mathrm{E}}$ & $F_{\mathrm{IS}}^{*}$ & $\begin{array}{l}\text { Size of focal } \\
\text { intron (bp) }\end{array}$ \\
\hline \multirow[t]{7}{*}{ Ck7 } & Siganus rivulatus (Siganidae) & 30 & 0 & 54 & - & - & - & - & - & - \\
\hline & Siganus luridus (Siganidae) & 30 & 0 & 54 & - & - & - & - & - & - \\
\hline & Thunnus obesus (Scombridae) & 100 & 1 & 45 & ToCk7 & Yes & 3 & - & - & 350 \\
\hline & Lates niloticus (Centropomidae) & 30 & 1 & 52 & $\operatorname{LnCk7}$ & Yes & - & - & - & - \\
\hline & Chaetodon citrinellus (Chaetodontidae) & 50 & multilocus & 54 & - & - & - & - & - & - \\
\hline & Chaetodon quadrimaculatus (=) & 50 & multilocus & 54 & - & - & - & - & - & - \\
\hline & Forcipiger flavissimus (=) & 50 & multilocus & 54 & - & - & - & - & - & - \\
\hline \multirow[t]{11}{*}{ Gpd2 } & Siganus rivulatus & 137 & 2 & 50 & SrGPD2-1 & Yes & 6 & 0.55 & n.s. & 250 \\
\hline & & & & & SrGPD2-2 & Yes & 5 & 0.32 & n.s. & 360 \\
\hline & Siganus luridus & 126 & 1 & 53 & SIGPD2 & Yes & 3 & 0.17 & n.s. & 175 \\
\hline & Ethmalosa fimbriata (Clupeidae) & 170 & 2 & 52 & EfGPD2-1 & Yes & 14 & 0.80 & n.s. & 200 \\
\hline & & & & & EfGPD2-2 & No & - & - & - & - \\
\hline & Chaetodon citrinellus & 50 & 1 & 54 & CcGPD2 & No & - & - & - & 350 \\
\hline & Chaetodon quadrimaculatus & 60 & 1 & 54 & $C q G P D 2$ & No & - & - & - & 350 \\
\hline & Forcipiger flavissimus & 60 & 1 & 54 & FfGPD2 & No & - & - & - & 350 \\
\hline & Dascyllus aruanus (Pomacentridae) & 60 & 1 & 54 & DaGPD2 & No & - & - & - & 350 \\
\hline & Pomacentrus pavo (=) & 60 & 1 & 54 & PpGPD2 & No & - & - & - & 350 \\
\hline & Plectroglyphidodon dickii (=) & 40 & 1 & 54 & PdGPD2 & No & - & - & - & 350 \\
\hline \multirow[t]{10}{*}{ GH2 } & Siganus rivulatus & 137 & 2 & 54 & SrGH2-1 & Yes & 2 & 0.23 & n.s. & 250 \\
\hline & & & & & $\mathrm{SrGH} 2-2$ & Yes & 4 & 0.61 & n.s. & 500 \\
\hline & Siganus luridus & 126 & 0 & 54 & - & - & - & - & - & - \\
\hline & Lates niloticus & 30 & 1 & 54 & $\mathrm{LnGH} 2$ & Yes & - & - & - & - \\
\hline & Chaetodon citrinellus & 50 & 1 & 54 & $\mathrm{CcGH} 2$ & No & - & - & - & 150 \\
\hline & Chaetodon quadrimaculatus & 60 & 1 & 54 & $\mathrm{CqGH} 2$ & No & - & - & - & 150 \\
\hline & Forcipiger flavissimus & 234 & 1 & 54 & FfGH2 & Yes & 12 & 0.72 & n.s. & 250 \\
\hline & Dascyllus aruanus & 60 & multilocus & 54 & - & - & - & - & - & - \\
\hline & Pomacentrus pavo & 60 & multilocus & 54 & - & - & - & - & - & - \\
\hline & Plectroglyphidodon dickii & 40 & multilocus & 54 & - & - & - & - & - & - \\
\hline \multirow[t]{10}{*}{ GH5 } & Siganus rivulatus & 137 & 1 & 52 & SrGH5 & No & - & - & - & 350 \\
\hline & Siganus luridus & 126 & 1 & 52 & SlGH5 & Yes & 4 & 0.34 & n.s. & 350 \\
\hline & Thunnus obesus & 20 & 1 & 52 & ToGH5 & Yes & 2 & - & - & - \\
\hline & Lates niloticus & 30 & multilocus & 52 & - & Yes & - & - & - & - \\
\hline & Chaetodon citrinellus & 198 & 1 & 54 & - & No & - & - & - & 300 \\
\hline & Chaetodon quadrimaculatus & 233 & 1 & 54 & - & No & - & - & - & 300 \\
\hline & Forcipiger flavissimus & 234 & 1 & 54 & - & - & - & - & - & - \\
\hline & Dascyllus aruanus & 250 & 1 & 54 & - & - & - & - & - & - \\
\hline & Pomacentrus pavo & 167 & 1 & 54 & PpGH5 & Yes & 2 & 0.31 & n.s. & 300 \\
\hline & Plectroglyphidodon dickii & 79 & 2 & 54 & $\begin{array}{l}\text { PdGH5-1 } \\
\text { пגרזт ? }\end{array}$ & No & - & - & - & 300 \\
\hline
\end{tabular}


Table 2 Continued

\begin{tabular}{|c|c|c|c|c|c|c|c|c|c|c|}
\hline Intron name & Species name & $\begin{array}{l}\text { Sample } \\
\text { size (ind.) }\end{array}$ & $\begin{array}{l}\text { No. of } \\
\text { amplified } \\
\text { loci }\end{array}$ & $\begin{array}{l}\text { Annealing } \\
\text { temp. }\left({ }^{\circ} \mathrm{C}\right)\end{array}$ & $\begin{array}{l}\text { Name of } \\
\text { amplified } \\
\text { loci }\end{array}$ & $\begin{array}{l}\text { Length } \\
\text { polymorphism }\end{array}$ & $\begin{array}{l}\text { No. of } \\
\text { alleles at } \\
\text { each locus }\end{array}$ & $H_{\mathrm{E}}$ & $F_{\mathrm{IS}}^{*}$ & $\begin{array}{l}\text { Size of focal } \\
\text { intron (bp)† }\end{array}$ \\
\hline \multirow[t]{3}{*}{ AldoC1 } & Siganus rivulatus & 137 & 1 & 53 & SrAldoC1 & Yes & 4 & 0.12 & n.s. & 230 \\
\hline & Siganus luridus & 126 & 1 & 54 & SlAldoC1 & Yes & 2 & 0.19 & n.s. & 240 \\
\hline & Dicentrarchus labrax (Moronidae) & 951 & 1 & 60 & DlAldoC1 & Yes & 3 & 0.78 & n.s. & 325 \\
\hline \multirow[t]{12}{*}{ AldoB1 $\ddagger$} & Siganus rivulatus & 137 & 1 & 49 & SrAldoB1 & Yes & 2 & 0.11 & n.s. & 320 \\
\hline & Siganus luridus & 126 & 3 & 52 & SlAldoB1-1 & Yes & 2 & 0.33 & n.s. & 175 \\
\hline & & & & & SlAldoB1-2 & Yes & 2 & 0.19 & n.s. & 200 \\
\hline & & & & & SlAldoB1-3 & Yes & 3 & 0.62 & n.s. & 400 \\
\hline & Chaetodon citrinellus & 36 & 1 & 54 & CcAldoB1 & No & - & - & - & - \\
\hline & Chaetodon quadrimaculatus & 10 & 1 & 54 & CqAldoB1 & No & - & - & - & - \\
\hline & Forcipiger flavissimus & 20 & 1 & 54 & FfAldoB1 & No & - & - & - & - \\
\hline & Dascyllus aruanus & 10 & 1 & 54 & DaAldoB1 & Yes & - & - & - & 300 \\
\hline & Pomacentrus pavo & 10 & 1 & 54 & PpAldoB1 & Yes & - & - & - & 300 \\
\hline & Plectroglyphidodon dickii & 10 & 1 & 54 & PdAldoB1 & Yes & - & - & - & 300 \\
\hline & Dicentrarchus labrax & 100 & 1 & 52 & DlAldoB1 & No & - & - & - & 262 \\
\hline & Decapterus russelli (Carangidae) & 458 & 1 & 52 & DrAldoB1 & Yes & 7 & 0.31 & n.s. & 265 \\
\hline \multirow[t]{13}{*}{ AldoB2 } & Siganus rivulatus & 20 & multilocus & 52 & - & - & - & - & - & - \\
\hline & Siganus luridus & 20 & multilocus & 52 & - & - & - & - & - & - \\
\hline & Sargocentron rubrum (Holocentridae) & 20 & multilocus & 50 & - & - & - & - & - & - \\
\hline & Upeneus moluccensis (Mullidae) & 20 & multilocus & 50 & - & - & - & - & - & - \\
\hline & Chaetodon citrinellus & 198 & 2 & 54 & CcAldoB2-1 & Yes & 4 & 0.10 & n.s. & 180 \\
\hline & & & & & CcAldoB2-2 & Yes & - & - & - & 300 \\
\hline & Chaetodon quadrimaculatus & 10 & 2 & 54 & CqAldoB2-1 & No & - & - & - & 180 \\
\hline & & & & & CqAldoB2-2 & No & - & - & - & 300 \\
\hline & Forcipiger flavissimus & 20 & 2 & 54 & FfAldoB2-1 & No & - & - & - & 280 \\
\hline & & & & & FfAldoB2-2 & No & - & - & - & 350 \\
\hline & Dascyllus aruanus & 250 & 1 & 54 & DaAldoB2 & Yes & 3 & 0.15 & n.s. & 200 \\
\hline & Pomacentrus pavo & 10 & 1 & 54 & PpAldoB2 & No & - & - & - & 200 \\
\hline & Plectroglyphidodon dickii & 10 & 1 & 54 & PdAldoB2 & No & - & - & - & 200 \\
\hline \multirow[t]{10}{*}{ AldoB4 } & Chaetodon citrinellus & 36 & 1 & 54 & CcAldoB4 & No & - & - & - & 200 \\
\hline & Chaetodon quadrimaculatus & 10 & 1 & 54 & CqAldoB4 & No & - & - & - & 200 \\
\hline & Forcipiger flavissimus & 20 & 1 & 54 & FfAldoB4 & No & - & - & - & 200 \\
\hline & Dascyllus aruanus & 255 & 2 & 54 & DaAldoB4-1 & Yes & 2 & 0.41 & n.s. & 200 \\
\hline & & & & & DaAldoB4-2 & No & - & - & - & 200 \\
\hline & Pomacentrus pavo & 167 & 2 & 54 & PpAldoB4-1 & Yes & 2 & 0.48 & n.s. & 200 \\
\hline & & & & & PpAldoB4-2 & No & - & - & - & 200 \\
\hline & Plectroglyphidodon dickii & 10 & 2 & 54 & PdAldoB4-1 & No & - & - & - & 200 \\
\hline & & & & & PdAldoB4-2 & No & - & - & - & 200 \\
\hline & Ethmalosa fimbriata & 68 & 1 & 50 & EfAldoB4 & Yes & 12 & 0.87 & n.s. & 380 \\
\hline
\end{tabular}


Table 2 Continued

\begin{tabular}{|c|c|c|c|c|c|c|c|c|c|c|}
\hline Intron name & Species name & $\begin{array}{l}\text { Sample } \\
\text { size (ind.) }\end{array}$ & $\begin{array}{l}\text { No. of } \\
\text { amplified } \\
\text { loci }\end{array}$ & $\begin{array}{l}\text { Annealing } \\
\text { temp. }\left({ }^{\circ} \mathrm{C}\right)\end{array}$ & $\begin{array}{l}\text { Name of } \\
\text { amplified } \\
\text { loci }\end{array}$ & $\begin{array}{l}\text { Length } \\
\text { polymorphism }\end{array}$ & $\begin{array}{l}\text { No. of } \\
\text { alleles at } \\
\text { each locus }\end{array}$ & $H_{\mathrm{E}}$ & $F_{\mathrm{IS}}^{*}$ & $\begin{array}{l}\text { Size of focal } \\
\text { intron (bp) } \dagger\end{array}$ \\
\hline \multirow[t]{7}{*}{ AldoB5 } & Siganus rivulatus & 20 & 0 & 58 & - & - & - & - & - & - \\
\hline & Siganus luridus & 20 & 0 & 58 & - & - & - & - & - & - \\
\hline & Sargocentron rubrum & 20 & 0 & 58 & - & - & - & - & - & - \\
\hline & Upeneus moluccensis & 20 & 0 & 58 & - & - & - & - & - & - \\
\hline & Chaetodon citrinellus & 36 & 1 & 54 & CcAldoB5 & No & - & - & - & 200 \\
\hline & Chaetodon quadrimaculatus & 10 & 1 & 54 & CqAldoB5 & No & - & - & - & 200 \\
\hline & Forcipiger flavissimus & 20 & 1 & 54 & FfAldoB5 & No & - & - & - & 200 \\
\hline \multirow[t]{11}{*}{ GnRH3-1 } & Siganus rivulatus & 136 & 2 & 48 & SrGnRH3-1-1 & Yes & 2 & 0.41 & n.s. & 250 \\
\hline & & & & & SrGnRH3-1-2 & Yes & 9 & 0.77 & n.s. & 400 \\
\hline & Siganus luridus & 126 & 3 & 48 & SlGnRH3-1-1 & Yes & 5 & 0.53 & n.s. & 275 \\
\hline & & & & & SlGnRH3-1-2 & Yes & 4 & 0.15 & n.s. & 325 \\
\hline & & & & & SlGnRH3-1-3 & Yes & 8 & 0.74 & n.s. & 375 \\
\hline & Chaetodon citrinellus & 198 & 1 & 54 & CcGnRH3-1 & Yes & 8 & 0.67 & 0.43 & 280 \\
\hline & Chaetodon quadrimaculatus & 233 & 1 & 54 & $\mathrm{CqGnRH} 3-1$ & Yes & 6 & 0.61 & n.s. & 280 \\
\hline & Forcipiger flavissimus & 50 & 1 & 54 & $F f G n R H 3-1$ & No & - & - & - & 280 \\
\hline & Dascyllus aruanus & 20 & 1 & 54 & $\mathrm{DaGnRH3-1}$ & No & - & - & - & 250 \\
\hline & Pomacentrus pavo & 20 & 1 & 54 & $P p G n R H 3-1$ & No & - & - & - & 250 \\
\hline & Plectroglyphidodon dickii & 20 & 0 & 54 & - & - & - & - & - & - \\
\hline \multirow[t]{9}{*}{ GnRH3-2 } & Siganus rivulatus & 137 & 1 & 56 & SrGnRH3-2 & No & - & - & - & 230 \\
\hline & Siganus luridus & 126 & 1 & 56 & SlGnRH3-2 & No & - & - & - & 230 \\
\hline & Decapterus russelli & 458 & 1 & 52 & DrGnRH3-2 & Yes & 7 & 0.71 & n.s. & 300 \\
\hline & Chaetodon citrinellus & 198 & 1 & 54 & $\mathrm{CcGnRH} 3-2$ & Yes & 4 & 0.52 & 0.21 & 280 \\
\hline & Chaetodon quadrimaculatus & 233 & 1 & 54 & $\mathrm{C} q \mathrm{GnRH} 3-2$ & Yes & 4 & 0.53 & n.s. & 280 \\
\hline & Forcipiger flavissimus & 234 & 1 & 54 & FfGnRH3-2 & Yes & 7 & 0.36 & n.s. & 300 \\
\hline & Dascyllus aruanus & 20 & 1 & 54 & $\mathrm{DaGnRH3-2}$ & No & - & - & - & 300 \\
\hline & Pomacentrus pavo & 20 & 1 & 54 & $P p G n R H 3-2$ & No & - & - & - & 300 \\
\hline & Plectroglyphidodon dickii & 79 & 1 & 50 & PdGnRH3-2 & Yes & 13 & 0.69 & n.s. & 300 \\
\hline \multirow[t]{9}{*}{ GnRH3-3 } & Siganus rivulatus & 137 & 1 & 52 & SrGnRH3-3 & Yes & 4 & 0.40 & n.s. & 325 \\
\hline & Siganus luridus & 126 & 1 & 53 & SlGnRH3-3 & Yes & 4 & 0.48 & n.s. & 375 \\
\hline & Thunnus obesus & 20 & 1 & 52 & ToGnRH3-3 & No & - & - & - & - \\
\hline & Chaetodon citrinellus & 50 & 1 & 54 & $\mathrm{CcGnRH} 3-3$ & No & - & - & - & 400 \\
\hline & Chaetodon quadrimaculatus & 550 & 1 & 54 & $\mathrm{CqGnRH3-3}$ & No & - & - & - & 400 \\
\hline & Forcipiger flavissimus & 50 & 1 & 54 & FfGnRH3-3 & No & - & - & - & 400 \\
\hline & Dascyllus aruanus & 20 & 1 & 54 & $\mathrm{DaGnRH} 3-3$ & Yes & - & - & - & 400 \\
\hline & Pomacentrus pavo & 20 & 1 & 54 & $P p G n R H 3-3$ & No & - & - & - & 400 \\
\hline & Plectroglyphidodon dickii & 20 & 1 & 54 & PdGnRH3-3 & No & - & - & - & 400 \\
\hline
\end{tabular}




\begin{tabular}{|c|c|c|c|c|c|c|c|c|c|c|}
\hline Intron name & Species name & $\begin{array}{l}\text { Sample } \\
\text { size (ind.) }\end{array}$ & $\begin{array}{l}\text { No. of } \\
\text { amplified } \\
\text { loci }\end{array}$ & $\begin{array}{l}\text { Annealing } \\
\text { temp. }\left({ }^{\circ} \mathrm{C}\right)\end{array}$ & $\begin{array}{l}\text { Name of } \\
\text { amplified } \\
\text { loci }\end{array}$ & $\begin{array}{l}\text { Length } \\
\text { polymorphism }\end{array}$ & $\begin{array}{l}\text { No. of } \\
\text { alleles at } \\
\text { each locus }\end{array}$ & $H_{\mathrm{E}}$ & $F_{\mathrm{IS}}^{*}$ & $\begin{array}{l}\text { Size of focal } \\
\text { intron (bp) } \dagger\end{array}$ \\
\hline \multirow[t]{7}{*}{ Am2B-1 } & Siganus rivulatus & 137 & 3 & 59 & SrAm2B-1-1 & Yes & 13 & 0.79 & 0.48 & 250 \\
\hline & & & & & SrAm2B-1-2 & No & - & - & - & 275 \\
\hline & & & & & SrAm2B-1-3 & Yes & 4 & 0.37 & n.s. & 400 \\
\hline & Siganus luridus & 126 & 2 & 59 & SlAm2B-1-1 & No & - & - & - & 275 \\
\hline & & & & & $S l A m 2 B-1-2$ & Yes & 4 & 0.46 & n.s. & 400 \\
\hline & Thunnus obesus & 20 & 1 & 48 & ToAm2B-1 & Yes & 2 & - & - & - \\
\hline & Lates niloticus & 30 & 1 & 52 & $\operatorname{Ln} A m 2 B-2$ & Yes & - & - & - & - \\
\hline \multirow[t]{3}{*}{ Am2B-2 } & Siganus rivulatus & 137 & 2 & 57 & SrAm2B-2-1 & Yes & 2 & 0.46 & n.s. & 350 \\
\hline & & & & & SrAm2B-2-2 & Yes & 4 & 0.54 & n.s. & 375 \\
\hline & Siganus luridus & 126 & multilocus & 57 & - & - & - & - & - & - \\
\hline \multirow[t]{2}{*}{ Am2B-3 } & Siganus rivulatus & 137 & 0 & 58 & - & - & - & - & - & - \\
\hline & Siganus luridus & 126 & 0 & 58 & - & - & - & - & - & - \\
\hline \multirow[t]{3}{*}{$\operatorname{Tr} 1$} & Siganus rivulatus & 137 & 1 & 50 & SrTr1 & Yes & 2 & 0.19 & n.s. & 250 \\
\hline & Siganus luridus & 126 & 1 & 50 & SlTr1 & Yes & 7 & 0.72 & n.s. & 300 \\
\hline & Ethmalosa fimbriata & 20 & multilocus & 45 & - & - & - & - & - & - \\
\hline \multirow[t]{4}{*}{ MhcII } & Siganus rivulatus & 20 & multilocus & 55 & - & Yes & - & - & - & - \\
\hline & Siganus luridus & 20 & 0 & 55 & - & - & - & - & - & - \\
\hline & Thunnus obesus & 20 & multilocus & 52 & - & - & - & - & - & - \\
\hline & Ethmalosa fimbriata & 20 & 0 & 52 & - & - & - & - & - & - \\
\hline
\end{tabular}

${ }^{*} F_{\mathrm{IS}}=\left(H_{\mathrm{E}}-H_{\mathrm{O}}\right) / H_{\mathrm{E}}$

†Approximate size.

$\ddagger$ Additionally, this intron was proven to amplify correctly in 23 other teleost species and one chondrichthyes species; (-) incomplete data; n.s. = insignificant value. 
of size between band systems was larger than the size polymorphism of each locus and, finally, (iii) we did not find significant departure from Hardy-Weinberg equilibrium in those samples in which we could analyse a sufficient number of individuals to test it properly.

\section{Acknowledgements}

We are grateful to N. Bierne, J.D. Durand, A. Rohfritsch, and J.F. Agnèse for their help.

\section{References}

Bierne N, Lehnert SA, Bédier E, Bonhomme F, Moore SS (2000) Screening for intron-length polymorphisms in penaeid shrimps using exon-primed intron-crossing (EPIC)-PCR. Molecular Ecology, 9, 233-235.

Côrte-Real HBSM, Dixon DR, Holland PWH (1994) Intron-targeted PCR: a new approach to survey neutral DNA polymorphism in bivalve populations. Marine Biology, 120, 407-413.

Daguin C, Bonhomme F, Borsa P (2001) The zone of sympatry and hybridization of Mytilus edulis and M. galloprovincialis, as described by intron length polymorphism at locus mac-1. Heredity, $86,342-354$.
Gomulski LM, Bourtzis K, Brogna S et al. (1998) Intron size polymorphism of the $A d h$ sub (1) gene parallels the worldwide colonization history of the Mediterranean fruit fly, Ceratitis capitata. Molecular Ecololgy, 7, 1729-1741.

He M, Haymer DS (1997) Polymorphic intron sequences detected within and between populations of the Oriental fruit fly (Diptera: Tephritidae). Annals of the Entomological Society of America, 90, 825-831.

He M, Haymer DS (1999) Genetic relationships of populations and the origins of new infestations of the Mediterranean fruit fly. Molecular Ecology, 8, 1247-1257.

Heslewood MM, Elphinstone MS, Tidemann SC, Baverstock PR (1998) Myoglobin intron variation in the Gouldian finch Erythrura gouldiae assessed by temperature gradient gel electrophoresis. Electrophoresis, 19, 142-151.

Lessa EP (1992) Rapid surveying of DNA sequence variation in natural populations. Molecular Biology and Evolution, 9, 323-330.

Palumbi SR (1995) Nucleic acids II: the polymerase chain reaction. In: Molecular Systematics, 2nd edn (ed. Hillis D, Moritz C), pp. 205247. Sinauer, Sunderland, MA.

Palumbi SR, Baker CS (1994) Contrasting population structure from nuclear intron sequences and mtDNA of humpback whales. Molecular Biology and Evolution, 11, 426-435.

Villablanca FX, Roderick GK, Palumbi SR (1998) Invasion genetics of the Mediterranean fruit fly: variation in multiple nuclear introns. Molecular Ecology, 7, 547-560. 